\title{
Balance Control and Knee Osteoarthritis Severity
}

\author{
Hee-Sang Kim, M.D., Dong Hwan Yun, M.D., Seung Don Yoo, M.D., Dong Hwan Kim, M.D., \\ Yong Seol Jeong, M.D., Jee-Sang Yun, M.D., Dae Gyu Hwang, M.D., Pil Kyo Jung, M.D., Seong He Choi, M.D.
}

Department of Rehabilitation Medicine \& Rehabilitation, Kyung Hee University College of Medicine, Seoul 130-702, Korea

Objective To investigate balance control according to the severity of knee osteoarthritis (OA) using clinical tests and Tetra-ataxiometric posturography $\left(\right.$ Tetrax $\left.^{\circledR}\right)$.

Method A total 80 patients with primary knee OA classified according to American College of Rheumatology criteria, and 40 age-matched controls were enrolled in this study. Of those with OA, 39 patients had mild OA (Kellgren-Lawrence [KL] grade 1,2) and the other 41 had moderate to severe OA (KL grade 3, 4). The postural control capabilities of the subjects were assessed using the timed up and go test (TUG), Berg balance scale (BBS), and Tetrax ${ }^{\circledR}$, which utilizes two paired force plates to measure vertical pressure fluctuations over both heels and forefeet. The subjects were checked for their stability index (ST), Fourier index, weight distribution index (WDI), and synchronization index (SI) in eight positions using Tetrax ${ }^{\circledR}$.

Results Patients with moderate to severe OA exhibited significantly higher stability indices in all positions than patients with mild OA. The Fourier index was also higher in patients with moderate to severe OA than in patients with mild OA. However, the weight distribution index and synchronization of both heels and forefeet were not significantly different in the three groups.

Conclusion These findings suggest that patients with moderate to severe OA have more deficits in balance control than those with mild disease. Therefore, evaluation of balance control and education aimed at preventing falls would be useful to patients with knee OA.

Key Words Knee osteoarthritis, Balance control, Posturography, Fall

Received September 3, 2010; Accepted August 1, 2011

Corresponding author: Seong He Choi

Department of Rehabilitation Medicine \& Rehibilitation, Kyung-hee University College of Medicine, Hoegi-dong, Dongdaemun-gu, Seoul 130-702, Korea

Tel: +82-2-958-8565, Fax: +82-2-958-8560, E-mail: 0624sung@naver.com

(a) This is an open-access article distributed under the terms of the Creative Commons Attribution Non-Commercial License (http:// creativecommons.org/licenses/by-nc/3.0) which permits unrestricted noncommercial use, distribution, and reproduction in any medium, provided the original work is properly cited.

Copyright $\odot 2011$ by Korean Academy of Rehabilitation Medicine

\section{INTRODUCTION}

Many aspects of the human body, such as the vestibular system, sight, proprioception, muscular strength, and cognition are related to the balance control, which is an important ability in everyday life. ${ }^{1}$ Knee osteoarthritis (OA) causes changes not only in the tissues within the articular cavity, but also the ligaments, tendons, and periarticular tissues including the muscles. ${ }^{2,3}$ It is already widely known that patients with knee OA have a disability in their proprioception compared to similar age controls. Also, the number of mechanical sensory receptors 
around the ligaments of knee joints with OA has been reported to be reduced when inspected histologically. ${ }^{4,5}$

The knee is the most commonly injured weight bearing joint, and OA of the knee is known to be a risk factor for fall injuries. ${ }^{6}$ Therefore, it would be valuable for OA patients to receive education related to preventing falls and rehabilitative training after evaluating their balance control abilities.

Clinical balance evaluation tests such as the timed up and go test (TUG), 10 meter walking test (10 m WT), functional reach test (FRT), ${ }^{7}$ and the Berg balance scale $(\mathrm{BBS})^{8,9}$ are all reported to have high intraclass correlation and reliability between test-retests. ${ }^{7-9}$ However, these evaluation tools may introduce a subjective element depending on the examiner or the examinee and they cannot assess all the various aspects of balance control. Recently, balance control ability has been evaluated using static or dynamic posturography, which can calculate balance control quantitatively by the postural sway, including how different postures, which induce change in sight and somatic sense, affect the pivot point of the body from front to back or from left to right. Specifically, the ability to control balance is analyzed quantitatively by calculating the difference in weight bearing distribution between the feet by using a force plate. ${ }^{10}$

The tetra-ataxiometric posturography system Tetrax ${ }^{\circledR}$ (Sunlight Medical Ltd., Ramat Gan, Israel) measures postural sway using the change in weight burden onto each of four separate force plates of the left and right anterior and posterior feet, thus producing various postural variable factors (Fig. 1). Studies which predicted the fall risk of patients with diabetic neuropathy or cervical spinal injuries have been conducted with this method. ${ }^{11,12}$ Lately, a study evaluating the balance ability of Parkinson's disease patients using Tetrax ${ }^{\circledR}$ was reported in Korea. ${ }^{13}$

A previous study comparing knee OA patients with sex and age-matched controls showed that pain and muscle strength affects postural sway as the quadriceps femoris muscle strength and proprioception decreased and postural sway increased in the knee OA group. ${ }^{1}$ Also, based on an evaluation of balance control ability using static posturography, the worse the radiologic test results were, the more increased the knee OA patients' postural sway became. $^{14}$

In another study comparing the peak extension moment of the knee joint in patients with a Kellgren-Lawrence (KL) grade $\leq 2$ and $K L$ grade $\geq 3$, the severity of knee OA correlated with the simple radiologic test results with regard to disease progression. ${ }^{15}$ Therefore, in our study, patients with a KL grade $\leq 2$ were labeled as the 'mild group' while patients with a KL grade $\geq 3$ were labeled as the 'moderate to severe group'. By evaluating several postural variables using Tetrax ${ }^{\circledR}$, we quantitatively compared the difference in balancing ability between the two OA groups and a control group of normal elderly patients of similar age.

\section{MATERIALS AND METHODS}

\section{Subjects}

A total of 80 knee OA patients over age 50 were chosen as subjects from among patients being treated at the Rehabilitation Center of our hospital during the period of
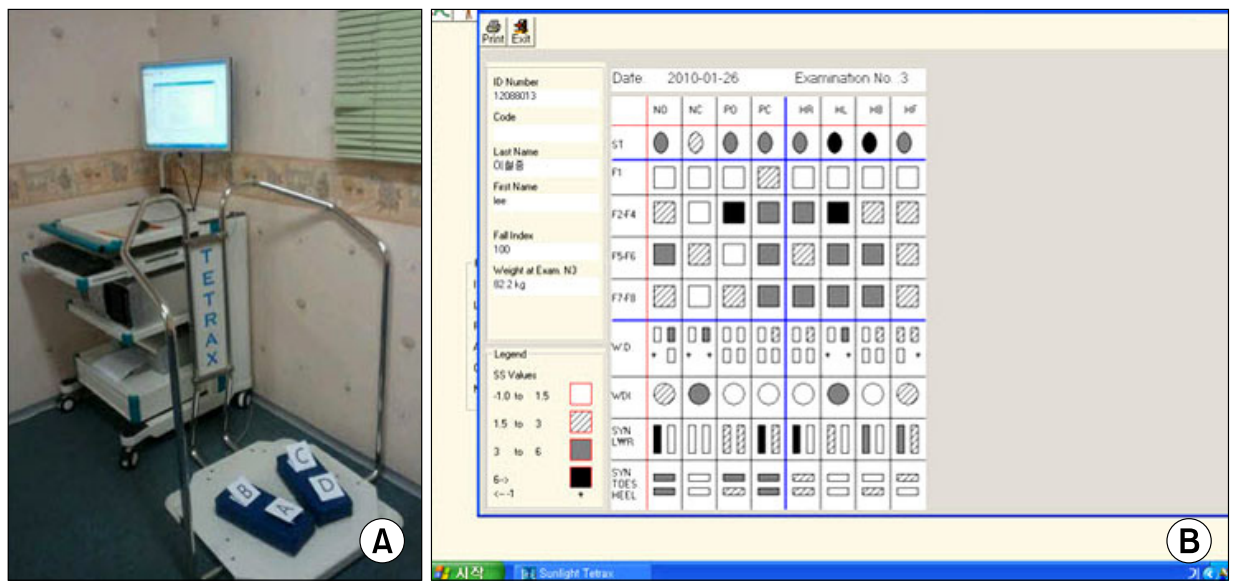

Fig. 1. Tetra-ataxiometric posturo graphy $\operatorname{Tetrax}^{\circledR}$ (A) postural stability in a patient with knee OA using $\operatorname{Tetrax}^{\circledR}(\mathrm{B})$ 
Table 1. General Characteristics of Subjects

\begin{tabular}{lccccc}
\hline & A $(\mathbf{n}=\mathbf{3 9})$ & $\mathbf{B}(\mathbf{n}=\mathbf{4 1})$ & $\mathbf{C}(\mathbf{n}=\mathbf{4 0})$ & F-value & $\mathbf{p}$ \\
\hline Age (years) & $62.9 \pm 6.1$ & $60.4 \pm 6.3$ & $60.3 \pm 3.9$ & 1.994 & 0.141 \\
Height $(\mathrm{cm})$ & $155.9 \pm 3.9$ & $157.1 \pm 4.8$ & $159.8 \pm 4.2$ & 0.398 & 0.530 \\
Weight $(\mathrm{kg})$ & $58.1 \pm 7.4$ & $59.6 \pm 7.4$ & $57.3 \pm 7.3$ & 0.811 & 0.447 \\
TUG (sec) & $11.0 \pm 1.7^{*^{\dagger}}$ & $8.4 \pm 0.9^{\dagger}$ & $8.0 \pm 1.2$ & 68.869 & 0.000 \\
BBS & $45.9 \pm 7.8^{*^{\dagger}}$ & $54.6 \pm 1.9^{\dagger}$ & $56.0 \pm 0.0$ & 43.446 & 0.000 \\
\hline
\end{tabular}

Values are mean \pm standard deviation

A: Moderate to severe osteoarthritis (OA), B: Mild OA, C: Controls, TUG: Timed up and go test, BBS: Berg balance scale ${ }^{*} \mathrm{p}<0.05$ between $\mathrm{A}$ and $\mathrm{B},{ }^{\dagger} \mathrm{p}<0.05$ between $\mathrm{A}$ and $\mathrm{C},{ }^{\dagger} \mathrm{p}<0.05$ between $\mathrm{B}$ and $\mathrm{C}$

Table 2. Testing Conditions for the Eight Posturographic Tests

\begin{tabular}{ccccl}
\hline Positions & Head position & Eyes & Ground & \multicolumn{1}{c}{ Purpose } \\
\hline NO & Neutral & Open & Solid & Neutral position \\
NC & Neutral & Closed & Solid & Elimination of visual system \\
PO & Neutral & Open & Elastic & Elimination of somatosensory system \\
PC & Neutral & Closed & Elastic & Elimination of somatosensory and visual systems \\
HR & Rotated to the right & Closed & Solid & Elimination of visual system and vestibular stress \\
HL & Rotated to the left & Closed & Solid & Elimination of visual system and vestibular stress \\
HB & Reclined & Closed & Solid & $\begin{array}{c}\text { Elimination of visual system, vestibular, and cervical } \\
\text { stress }\end{array}$ \\
& Inclined & Closed & Solid & $\begin{array}{c}\text { Elimination of visual system, vestibular, and cervical } \\
\text { HF }\end{array}$ \\
& & & & stress \\
\hline
\end{tabular}

June, 2010 through May, 2011. The American College of Rheumatology criteria was used to diagnose knee OA. ${ }^{16}$ Patients with unilateral knee OA were included and patients with bilateral involvement but with different KL grades were chosen for the more severe OA group. Patients with cerebrovascular disease, disease of the vestibular apparatus, severe visual disturbance, radiculopathy, peripheral neuropathy, impaired cognition (mini-mental status examination $<24$ ), psychiatric disease, or dismemberment were excluded from the study. The control group was comprised of healthy adults or those over the age of 50 that were capable of independent ambulation. Those that had any neurologic disease, musculoskeletal abnormality, internal disease, or medication that affects balance control were excluded. In addition, those without any pain or stiffness in the knee but having crepitus and swelling were also excluded, leaving a total of 40 subjects (Table 1) who agreed to participate after sufficient explanation of the study.

\section{Methods}

Both the knee OA case group and the control group had their weight and height measured, and information of all past medical histories such as internal disease were attained through surveys and from medical data prior to testing. Balance control ability was evaluated by using both the clinical assessment method and posturography.

Clinical assessment: (1) TUG test: Subjects sat in a normal armchair with arms draped over the armrest. At the signal to start, participants got up, walked three meters at ordinary speed, turned around, and came back and sat back down in the chair. An examiner used a stopwatch to time the whole procedure from the point of getting up from the chair to the point of sitting back down. A time within 10 seconds was considered normal, and time over 14 seconds was suspected as a deterioration of balance control. ${ }^{17}$

(2) BBS: The three categories of sitting, standing, and postural change were evaluated in 14 areas with each area having a low score of 0 points and a high score of 4 points, for a possible total of 56 points with a higher score 


\section{implying better balance control., ${ }^{9,18}$}

Assessment using posturography: Static posturography was conducted using Tetrax ${ }^{\circledR}$ (Sunlight Medical Ltd., Ramat Gan, Israel). The force plate of Tetrax ${ }^{\circledR}$ is formed with four separate plates and each force plate measures the perpendicular pressure of the anterior and posterior feet. The anterior feet force plates (left force plate: B, right force plate: D) are rectangles of $12 \times 19 \mathrm{~cm}$, and the posterior feet force plates (left force plate: A, right force plate: C) are squares of $12 \times 12 \mathrm{~cm}$ (Fig. 1-A). The data obtained by the pressure burden of each force plate when the subject stood on them was amplified and filtered, passed on to the computer, and analyzed by the Tetrax ${ }^{\circledR}$ software program (Fig. 1-B).

Subjects took off their shoes, positioned their feet on the plates, and stood in a comfortable position. Eight different postures were evaluated for the test and each posture was measured for 32 seconds (Table 2). The first posture, normal eye open (NO), was evaluated followed by normal eye close (NC) posture to limit the effect of eyesight, thus emphasizing the effect of somatic sense or the vestibular organ. Next, four different postures were tested in the following order - head right (HR), rotating the head $45^{\circ}$ to the right; head left (HL), rotating the head $45^{\circ}$ to the left; head backward (HB), tilting the head back $30^{\circ}$ and facing the ceiling; and head forward (HF), bending the head forward $30^{\circ}$ and facing the floor. These positions are sensitive to postural deviations in cases of problems with the vestibular apparatus, or the lumbar or cervical vertebrae. Finally, with a blue foamrubber pillow (Fig. 1-A) on the floor of the machine, pillow with eye open (PO) posture and pillow with eye close (PC) posture were rated facing front. The foamrubber pillow in the PO posture limits the interference of somatic senses, stressing the effect of eyesight in maintaining stability. Thus, a decrease of stability in this posture usually implies an abnormality in eyesight. The PC posture limits the input of both the visual and somatic senses, thereby putting stress on the vestibular organ. A decrease of stability in the PC posture indicates a problem with the vestibular organ. For the postural variable factors, the stability index (ST), Fourier index, weight distribution index (WDI), and the synchronization index (SI) were measured.

(1) Stability index (ST): The ST is a variable that indicates the degree of postural sway, which therefore tests the overall stability and ability to control and compensate for changes in posture. The higher the index score, the more unstable the posture.

(2) Weight distribution index (WDI): The WDI reflects the level of weight distributed on the four force plates, with the normal index being 4 to 6 . The normal percentage of weight put on each of the four force plates is $25 \%$. A high percentage points to a pathologic state where the weight distribution has changed radically from the physiologic $25 \%$.

(3) Fourier index: The Fourier index is a regression analysis of the postural sway intensity through Fourier transform, which shows a different frequency for each lesion that causes instability. A higher index for the frequency indicates a larger instability. Fluctuation at a low to medium frequency (F2-4) of 0.1-0.5 Hz usually signifies an abnormality in the vestibular organ or fatigue of the musculoskeletal system or drinking, and has a high index. Fluctuation at a medium to high frequency (F5-6) of 0.5-1 Hz shows a high index when the subject has an impairment in the somatic sensory response due to a decline in motor functions of the spine and lower extremities. Fluctuation at a high frequency (F7-8) of more than $1 \mathrm{~Hz}$, shows a high index when a postural instability occurs due to a central nervous system abnormality. ${ }^{19}$

(4) Synchronization index (SI): By comparing two of the oscillation waves measured from the body vibrations of the four force plates of $\mathrm{A}, \mathrm{B}, \mathrm{C}$, and $\mathrm{D}$, six combinations (AB, CD, BD, AC, BC, AD) can be derived (Fig. 1-A). The values span from $-1,000$ to 1,000 . A normal subject has an absolute value of 700 or more while a lower absolute value implies a decline in the fine posture-controlling ability of the feet. In this study, the movements between the anterior and posterior feet (force plates $A B, C D$ ), between the left and right anterior feet (force plates $\mathrm{BD}$ ), and between the left and right posterior feet (force plates AC) were observed. ${ }^{13,20}$

SPSS version 13.0 was used for the statistical analysis. The balance control abilities obtained from conventional data, BBS, TUG test, and Tetrax ${ }^{\circledR}$ of the mild and moderate to severe patient group and the control group, were analyzed. The Bonferroni method was used as a post-hoc test for multiple comparisons. If the p-value was $<0.05$, the result was considered to be statistically significant. 


\section{RESULTS}

\section{Clinical assessment}

The BBS of the control group was a perfect score of 56, and the TUG and BBS test results of the knee OA mild and moderate to severe patient groups showed a statistically significant difference compared to the control group
(Table 1).

\section{Assessment using posturography}

Stability index (ST): The moderate to severe group had a significantly higher $\mathrm{p}$-value $(\mathrm{p}<0.05)$ than the mild group and the control group. The higher ST in the moderate to severe group indicated that the general postural balance

Table 3. Comparison of Stability and Weight Distribution Indices in Eight Positions in Mild OA, Moderate to Severe OA, and Controls

\begin{tabular}{|c|c|c|c|c|c|c|c|c|c|c|}
\hline \multirow{2}{*}{ Position } & \multicolumn{5}{|c|}{ Stability index } & \multicolumn{5}{|c|}{ Weight distribution index } \\
\hline & A & B & $\mathrm{C}$ & F-value & $\mathbf{p}$ & A & B & $\mathbf{C}$ & F-value & $\mathbf{p}$ \\
\hline NO & $18.2 \pm 6.4^{* \dagger}$ & $14.9 \pm 4.5$ & $15.0 \pm 7.0$ & 3.804 & 0.026 & $6.6 \pm 4.2$ & $5.4 \pm 3.7$ & $5.1 \pm 2.9$ & 1.578 & 0.211 \\
\hline NC & $25.4 \pm 11.1^{* \dagger}$ & $19.5 \pm 6.2$ & $17.0 \pm 7.1$ & 8.630 & 0.000 & $6.8 \pm 4.5$ & $5.6 \pm 3.4$ & $5.2 \pm 2.1$ & 2.105 & 0.127 \\
\hline $\mathrm{PO}$ & $24.3 \pm 9.3^{* \dagger}$ & $19.4 \pm 6.0$ & $18.9 \pm 7.7$ & 9.209 & 0.000 & $6.1 \pm 4.1$ & $5.9 \pm 3.7$ & $5.7 \pm 3.6$ & 1.994 & 0.141 \\
\hline $\mathrm{PC}$ & $36.0 \pm 15.1^{* \dagger}$ & $28.0 \pm 7.1$ & $22.9 \pm 7.9$ & 11.442 & 0.000 & $6.5 \pm 4.8$ & $6.0 \pm 3.8$ & $5.9 \pm 3.4$ & 1.229 & 0.297 \\
\hline HR & $21.6 \pm 8.9^{* \dagger}$ & $15.3 \pm 3.8$ & $15.2 \pm 6.4$ & 10.024 & 0.000 & $6.6 \pm 4.0$ & $5.4 \pm 3.2$ & $4.2 \pm 2.1$ & 2.502 & 0.087 \\
\hline HL & $22.1 \pm 10.0^{* \dagger}$ & $15.9 \pm 4.6$ & $15.5 \pm 6.9$ & 7.647 & 0.001 & $5.8 \pm 3.8$ & $5.1 \pm 2.6$ & $4.9 \pm 2.4$ & 1.349 & 0.264 \\
\hline HB & $24.6 \pm 9.9^{* \dagger}$ & $17.4 \pm 4.9$ & $17.3 \pm 7.9$ & 8.576 & 0.000 & $6.0 \pm 4.2$ & $5.4 \pm 2.7$ & $5.3 \pm 3.2$ & 1.109 & 0.334 \\
\hline $\mathrm{HF}$ & $24.1 \pm 13.4^{* \dagger}$ & $16.0 \pm 4.7$ & $15.6 \pm 6.9$ & 7.626 & 0.001 & $5.9 \pm 3.8$ & $5.1 \pm 2.3$ & $4.1 \pm 1.8$ & 2.625 & 0.770 \\
\hline
\end{tabular}

Values are mean \pm standard deviation

A: Moderate to severe osteoarthritis (OA), B: Mild OA, C: Controls, NO: Normal position with eyes open, NC: Normal position with eyes closed, PO: Eyes open on pillows, PC: Eyes closed on pillows, HR: Head turned right and eyes closed, HL: Head turned left and eyes closed, HB: Eyes closed raising head backward $30^{\circ}$, HF: Eyes closed with head forward approximately $30^{\circ}$

${ }^{*} \mathrm{p}<0.05$ between $\mathrm{A}$ and $\mathrm{B},{ }^{\dagger} \mathrm{p}<0.05$ between $\mathrm{A}$ and $\mathrm{C}$

Table 4. Comparison of Fourier Indices in Eight Positions in Mild OA, Moderate to Severe OA, and Controls

\begin{tabular}{|c|c|c|c|c|c|c|c|c|c|c|c|c|}
\hline \multirow[t]{2}{*}{ Position } & \multicolumn{4}{|c|}{$\begin{array}{c}\text { Lower medium frequency } \\
(0.1-0.5 \mathrm{~Hz}, \mathrm{~F} 2-4)\end{array}$} & \multicolumn{4}{|c|}{$\begin{array}{c}\text { Higher medium frequency } \\
(0.5-1.0 \mathrm{~Hz}, \mathrm{~F} 5-6)\end{array}$} & \multicolumn{4}{|c|}{$\begin{array}{l}\text { High frequency } \\
(>1.0 \mathrm{~Hz}, \mathrm{~F} 7-8)\end{array}$} \\
\hline & A & B & C & $\mathbf{p}$ & A & B & C & p & A & B & C & $\mathbf{p}$ \\
\hline NO & $24.0 \pm 10.3$ & $23.4 \pm 7.3$ & $23.5 \pm 10.9$ & 0.248 & $6.6 \pm 2.7$ & $5.7 \pm 2.2$ & $6.8 \pm 5.8$ & 0.435 & $1.1 \pm 0.4$ & $1.0 \pm 0.3$ & $1.1 \pm 0.9$ & 0.413 \\
\hline NC & $34.2 \pm 11.8^{* \dagger}$ & $27.7 \pm 7.9$ & $25.4 \pm 9.2$ & 0.001 & $9.6 \pm 4.6^{\dagger}$ & $8.4 \pm 6.8$ & $5.8 \pm 2.8$ & 0.021 & $1.5 \pm 0.8^{* \dagger}$ & $1.2 \pm 0.4$ & $1.0 \pm 0.4$ & 0.001 \\
\hline $\mathrm{PO}$ & $29.6 \pm 10.2$ & $25.1 \pm 8.9$ & $24.5 \pm 9.9$ & 0.052 & $8.9 \pm 3.9^{* \dagger}$ & $7.0 \pm 2.2$ & $7.3 \pm 2.3$ & 0.016 & $1.6 \pm 0.7^{* \dagger}$ & $1.3 \pm 0.5$ & $1.3 \pm 0.7$ & 0.004 \\
\hline PC & $42.9 \pm 11.6$ & $36.6 \pm 10.8$ & $30.5 \pm 10.9$ & 0.780 & $12.6 \pm 5.6^{* \dagger}$ & $10.1 \pm 3.5$ & $8.2 \pm 3.6$ & 0.000 & $2.3 \pm 1.0^{* \dagger}$ & $1.8 \pm 0.5$ & $1.4 \pm 0.5$ & 0.000 \\
\hline HR & $27.5 \pm 8.1$ & $20.8 \pm 6.1$ & $22.9 \pm 8.4$ & 0.144 & $8.3 \pm 3.5^{* \dagger}$ & $6.0 \pm 1.7$ & $6.0 \pm 2.6$ & 0.000 & $1.5 \pm 0.6^{* \dagger}$ & $1.0 \pm 0.3$ & $1.0 \pm 0.5$ & 0.000 \\
\hline HL & $29.2 \pm 11.3$ & $21.0 \pm 5.9$ & $23.1 \pm 9.1$ & 0.130 & $8.6 \pm 4.2^{* \dagger}$ & $6.2 \pm 2.1$ & $6.1 \pm 3.4$ & 0.006 & $1.6 \pm 0.7^{* \dagger}$ & $1.1 \pm 0.4$ & $1.0 \pm 0.6$ & 0.000 \\
\hline HB & $32.3 \pm 11.0^{* \dagger}$ & $25.8 \pm 7.1$ & $24.6 \pm 11.0$ & 0.011 & $8.6 \pm 3.1^{* \dagger}$ & $6.3 \pm 2.1$ & $6.2 \pm 3.9$ & 0.003 & $1.9 \pm 0.8^{* \dagger}$ & $1.2 \pm 0.4$ & $1.1 \pm 0.6$ & 0.000 \\
\hline HF & $33.0 \pm 10.8^{* \dagger}$ & $22.3 \pm 6.9$ & $21.3 \pm 9.4$ & 0.000 & $9.1 \pm 5.2^{*^{\dagger}}$ & $6.3 \pm 2.0$ & $6.2 \pm 3.0$ & 0.004 & $1.8 \pm 1.1^{* \dagger}$ & $1.1 \pm 0.4$ & $1.0 \pm 0.4$ & 0.000 \\
\hline
\end{tabular}

Values are mean \pm standard deviation

A: Moderate to severe osteoarthritis (OA), B: Mild OA, C: Controls, NO: Normal position with eyes open, NC: Normal position with eyes closed, PO: Eyes open on pillows, PC: Eyes closed on pillows, HR: Head turned right and eyes closed, HL: Head turned left and eyes closed, HB: Eyes closed with raising head backward $30^{\circ}$, HF: Eyes closed with head forward approximately $30^{\circ}$

${ }^{*} \mathrm{p}<0.05$ between $\mathrm{A}$ and $\mathrm{B},{ }^{\dagger} \mathrm{p}<0.05$ between $\mathrm{A}$ and $\mathrm{C}$ 
control was diminished in these patients (Table 3 ).

Weight distribution index (WDI): The WDI of the mild group, moderate to severe group, and control group did not show a statistically significant difference and each group remained within the normal value limits (Table 3 ). Fourier index: The moderate to severe group of knee OA patients had a significantly higher index value than the control group in the NC, $\mathrm{HB}$, and HF posture of F2-4; and NC, PO, PC, HR, HL, HB, and HF posture of F5-6 and F78. The moderate to severe group had a significantly higher index value than the mild group in the $\mathrm{NC}, \mathrm{HB}$, and $\mathrm{HF}$ postures of $\mathrm{F} 2-4$; PO, PC, HR, HL, HB, and HF postures in F5-6; and NC, PO, PC, HR, HL, HB, and HF postures of F7-8 (Table 4).

Synchronization index (SI): The only case where the SI values of the mild group and the moderate to severe group were significantly different was when CD (right anterior and posterior feet) and BD (right and left anterior feet) were compared in the NC position. In general, there was no statistically significant difference in the

Table 5. Comparison of Synchronization Indices in Eight Positions in Mild OA, Moderate to Severe OA, and Controls

\begin{tabular}{|c|c|c|c|c|}
\hline & $\mathrm{AB}$ & $\mathrm{CD}$ & $\mathrm{AC}$ & $\mathrm{BD}$ \\
\hline NOA & $745.2 \pm 206.3$ & $720.8 \pm 262.8$ & $659.3 \pm 261.4$ & $692.7 \pm 346.6$ \\
\hline B & $758.4 \pm 233.1$ & $728.1 \pm 201.1$ & $666.9 \pm 273.9$ & $721.0 \pm 258.9$ \\
\hline $\mathrm{C}$ & $761.8 \pm 228.2$ & $729.4 \pm 161.8$ & $663.9 \pm 314.1$ & $765.2 \pm 292.6$ \\
\hline $\mathrm{NCA}$ & $800.3 \pm 197.6$ & $705.6 \pm 247.4^{*}$ & $696.0 \pm 216.4$ & $697.2 \pm 282.1^{*}$ \\
\hline B & $821.9 \pm 222.7$ & $791.4 \pm 89.3$ & $736.5 \pm 235.9$ & $840.1 \pm 156.0$ \\
\hline $\mathrm{C}$ & $822.1 \pm 188.9$ & $795.3 \pm 174.2$ & $742.5 \pm 237.5$ & $848.9 \pm 223.5$ \\
\hline PO A & $685.2 \pm 281.0$ & $685.8 \pm 309.7$ & $745.7 \pm 270.3$ & $529.6 \pm 437.7$ \\
\hline B & $705.0 \pm 196.1$ & $693.7 \pm 181.0$ & $731.4 \pm 170.1$ & $532.8 \pm 282.9$ \\
\hline C & $675.3 \pm 299.7$ & $711.9 \pm 228.1$ & $693.6 \pm 226.9$ & $544.9 \pm 398.0$ \\
\hline PC A & $710.5 \pm 277.0$ & $824.1 \pm 292.4$ & $739.2 \pm 267.0$ & $759.8 \pm 380.4$ \\
\hline B & $827.8 \pm 186.2$ & $853.0 \pm 93.0$ & $817.2 \pm 175.3$ & $764.7 \pm 156.9$ \\
\hline C & $665.4 \pm 293.2$ & $895.2 \pm 253.5$ & $795.5 \pm 209.4$ & $777.1 \pm 373.2$ \\
\hline HR A & $722.1 \pm 298.7$ & $715.3 \pm 339.1$ & $605.4 \pm 303.2$ & $651.1 \pm 323.0$ \\
\hline B & $769.5 \pm 227.9$ & $785.3 \pm 237.7$ & $645.6 \pm 265.8$ & $728.2 \pm 282.7$ \\
\hline $\mathrm{C}$ & $738.7 \pm 275.2$ & $790.6 \pm 200.9$ & $518.2 \pm 357.7$ & $688.1 \pm 246.8$ \\
\hline HL A & $732.6 \pm 234.0$ & $727.0 \pm 775.8$ & $591.2 \pm 372.9$ & $663.7 \pm 267.2$ \\
\hline B & $754.6 \pm 234.0$ & $775.8 \pm 238.5$ & $576.8 \pm 308.3$ & $736.4 \pm 267.0$ \\
\hline $\mathrm{C}$ & $751.5 \pm 264.7$ & $761.4 \pm 268.6$ & $534.9 \pm 339.5$ & $737.7 \pm 183.9$ \\
\hline HB A & $805.5 \pm 185.8$ & $820.6 \pm 217.1$ & $696.9 \pm 302.1$ & $764.3 \pm 306.6$ \\
\hline B & $842.9 \pm 144.4$ & $848.6 \pm 162.4$ & $689.5 \pm 220.9$ & $769.3 \pm 132.5$ \\
\hline C & $826.1 \pm 201.0$ & $835.5 \pm 194.1$ & $698.6 \pm 261.0$ & $779.0 \pm 234.1$ \\
\hline HF A & $796.3 \pm 267.6$ & $807.6 \pm 261.2$ & $729.7 \pm 312.3$ & $821.6 \pm 318.6$ \\
\hline B & $815.3 \pm 80.3$ & $811.5 \pm 158.7$ & $730.8 \pm 180.2$ & $844.5 \pm 130.0$ \\
\hline $\mathrm{C}$ & $819.3 \pm 171.3$ & $812.5 \pm 174.6$ & $758.0 \pm 305.0$ & $837.7 \pm 274.5$ \\
\hline
\end{tabular}

Values are mean \pm standard deviation

Values of $\mathrm{AB}$ and $\mathrm{CD}$ are negative. Values of AC and BD are positive

AB: Synchronization index (SI) between heel and toe plates of left foot, CD: SI between heel and toe plates of right foot, AC: SI in both heel plates, BD: SI in both toe plates, A: Moderate to severe osteoarthritis (OA), B: Mild OA, C: Control, NO: Normal position with eyes open, NC: Normal position with eyes closed, PO: eyes open on pillows, PC: eyes closed on pillows, HR: Head turned right and eyes closed, HL: Head turned left and eyes closed, HB: Eyes closed raising head backward by $30^{\circ}$, HF: eyes closed with head forward approximately $30^{\circ}$

${ }^{*} \mathrm{p}<0.05$ between $\mathrm{A}$ and $\mathrm{B}$ 
index values of the combination of movement in the left and right anterior and posterior feet between the two groups (Table 5).

\section{DISCUSSION}

Knee OA is a factor that lowers the quality of life in the elderly. ${ }^{14}$ The prevalence of knee OA in the elderly varies from $26 \%$ to $63 \%$ depending on age and sex. ${ }^{21}$ Considering that our society is rapidly changing into an aging society, the complications arising from the deterioration of balance control ability associated with knee OA will continue to be a point of interest.

In particular, the decline of balance control can lead to fall injuries which result in serious physical, psychological, and social loss. As such, many studies concerning the prevention of falls and loss of balance control are in progress. ${ }^{22,23}$ An accurate evaluation of balance control in knee OA patients is necessary before further treatment can be recommended or developed. BBS and TUG tests are widely used as clinical assessment tools of balance control. These methods are easy to use by anyone and are reported to have high reliability between examiners and test-retests. ${ }^{7-9}$ We were able to confirm that these tools were clinically useful for rating balance control by proving a statistically significant difference between mild and moderate to severe OA groups and a control group. However, these clinical tests may allow for interference of subjective factors and may be difficult to apply with certain degrees of balance control loss.

When we compared the balance control ability of the mild, and moderate to severe OA groups to the control group using Tetrax ${ }^{\circledR}$, we were able to discover several points of difference. The ST, which quantitatively measures the postural sway according to changes in the pivoting center, was higher in all the postures of the moderate to severe group compared to those of the mild or control groups. The Fourier index showed a larger instability of posture in the moderate to severe group than the mild or control groups in all postures except the F5-6 and F7-8. Therefore, we can deduce that moderate to severe knee OA patients have a tendency to depend on their eyesight in order to compensate for their postural instability. Since all patients with central nervous system or vestibular organ abnormalities had been excluded, we can conclude that the decrease in balance control of the moderate to severe group was due to musculoskeletal fatigue, and a decline in motor functions of the spine and lower extremities. Therefore, this study indicates that not only treatment for OA but also training to prevent falls is necessary in patients with progressive knee OA. Also, the risk of fall injuries increases when patients close their eyes or walk on unstable ground surfaces, or when various head movements are required. Thus, in the home of knee OA patients, it is advised to keep the floor sound and even, avoid hanging signs and clocks too high, and keep necessary things in places requiring minimum head movement. With regard to balance control, training in various postures in a space with a floor made of a material that can stimulate diminished proprioception, and warnings that different head movements can cause fall injuries should be included.

Also in this study, in all postures except the one with the eyes closed, the weight distribution of the anterior and posterior part of both feet was comparatively even, and the combined movement between the anterior and posterior feet (force plates $\mathrm{AB}, \mathrm{CD}$ ), between the left and right anterior feet (force plate $\mathrm{BD}$ ), and between the left and right posterior feet (force plate AC) was in harmony, thus, even though there may be an increase in postural disturbance in moderate to severe OA patients, the body retains its ability to achieve balance.

The limits of this study are, first, there are not a large number of patients included although we expect that we can abstract more meaningful results if we replenish the number of patients and reanalyze the data in the future. Second, we did not perform radiologic tests of the knee in the control group. However, since we excluded any subjects with crepitus or swelling in the knee, it is unlikely that related confounders had any effect on the study. Third, since Tetrax ${ }^{\circledR}$ usually evaluates stability in the static condition, it is difficult to assess the problems of balance control in the dynamic state. Fourth, a study checking to see if the Tetrax ${ }^{\circledR}$ balance control index is confounded by age, weight, or height is lacking. However, any confounding effects of age, weight, or height are predicted to be minimal since age, weight, and height were not significantly different between the groups. In the future, a study confirming that the output of the Tetrax ${ }^{\circledR}$ balance assessment tool is not affected by age, weight, or height is needed. Fifth, we observed that the results of the Tetrax ${ }^{\circledR}$ test were affected when the subject did not 
comply with the examiner's orders or attend the test with a voluntary attitude. In particular, in the HR posture with eyes closed and head rotated $45^{\circ}$ to the right, HL posture with the head rotated $45^{\circ}$ to the left, HB posture with the head tilted back $30^{\circ}$, and HF posture with the head bowed forward $30^{\circ}$, subjects must be educated and understand accurately the position requirements.

\section{CONCLUSION}

This study included 80 knee OA patients divided into two groups. Those with a KL grade $\leq 2$ were labeled the 'mild group' and those with a KL grade $\geq 3$ were labeled the 'moderate to severe group'. All subjects were evaluated for balance control ability to determine if there was a correlation with OA severity.

We observed that moderate to severe OA patients had diminished balance control compared to mild OA patients and we were able to deduce that a decrease in muscle strength, proprioception, and increased pain contributes to postural instability. Quantitative evaluation using force plates with knee OA patients can be complemented with clinical balance control tests. Also, damage as the result of future fall injuries can be prevented if rehabilitation treatment and education as to the importance of preventing falls is enforced based upon the results of balance tests.

\section{REFERENCES}

1. Hassan BS, Mockett S, Doherty M. Static postural sway, proprioception, and maximal voluntary quadriceps contraction in patients with knee osteoarthritis and normal control subjects. Ann Rheum Dis 2001; 60: 612-618

2. Brandt KD, Dieppe P, Radin EL. Etiopathogenesis of osteoarthritis. Rheum Dis Clin North Am 2008; 34: 531-539

3. Martel-Pelletier J. Pathophysiology of osteoarthritis. Osteoarthritis Cartilage 1998; 6: 374-376

4. Barrett DS, Cobb AG, Bentley G. Joint proprioception in normal, osteoarthritic and replaced knees. J Bone Joint Surg Br 1991; 73: 53-56

5. Marks R, Quinney HA, Wessel J. Proprioceptive sensibility in women with normal and osteoarthritic knee joints. Clin Rheumatol 1993; 12: 170-175
6. Pandya NK, Draganich LF, Mauer A, Piotrowski GA, Pottenger L. Osteoarthritis of the knees increases the propensity to trip on an obstacle. Clin Orthop Relat Res 2005; 431: 150-156

7. Lee KM, Kang JW, Han SH. Evaluation of standing balance in hemiplegic patients using the functional reach test. J Korean Acad Rehab Med 2002; 26: 647651

8. Lee JJ, Lee HJ, Park JH, Han EY, Kim MJ, Jung HY. The Korean version of berg balance scale as an index of activity related to ambulation in subjects with stroke. J Korean Acad Rehab Med 2007; 31: 400-403

9. Jung HY, Park JH, Shim JJ, Kim MJ, Hwang MR, Kim SH. Reliability test of Korean version of berg balance scale. J Korean Acad Rehab Med 2006; 30: 611-618

10. Hermodsson Y, Ekdahl C, Persson BM, Roxendal G. Standing balance in trans-tibial amputees following vascular disease or trauma: a comparative study with healthy subjects. Prosthet Orthot Int 1994; 18: 150-158 11. Oppenheim U, Kohen-Raz R, Alex D, Kohen-Raz A, Azarya M. Postural characteristics of diabetic neuropathy. Diabetic Care 1999; 22: 328-332

12. Dehner C, Heym B, Maier D, Sander S, Arand M, Elbel M, Hartwig E, Kramer M. Postural control deficit in acute QTF grade whiplash injuries. Gait Posture 2008; 28: 113-119

13. Kim BR, Choi KH, Chun MH, Lee MC, Chung SJ, Jang KW. Evaluation of balance control in patients with idiopathic Parkinson's disease using tetra-ataxiometric posturography. J Korean Acad Rehab Med 2009; 33: 538-546

14. Masui T, Hasegawa Y, Yamaguchi J, Kanoh T, Ishiguro $\mathrm{N}$, Suzuki S. Increasing postural sway in rural-community-dwelling elderly persons with knee osteoarthritis. J Orthop Sci 2006; 11: 353-358

15. Thorp LE, Sumner DR, Block JA, Moisio KC, Shott S, Wimmer MA. Knee joint loading differs in individuals with mild compared with moderate medial knee osteoarthritis. Arthritis Rheum 2006; 54: 3842-3849

16. Altman R, Asch E, Bloch D, Bole G, Borenstein D, Brandt K, Christy W, Cooke TD, Greenwald R, Hochberg M, et al. Development of criteria for the classification and reporting of osteoarthritis. Classification of osteoarhritis of the knee. Diagnostic and Therapeutic Criteria Committee of the American Rheumatism Association. Arthritis Rheum 1986; 29: 1039-1049 
17. Shumway-Cook A, Brauser S, Woollacott M. Predicting the probability for falls in community-dwelling older adults using the Timed Up \& Go Test. Phys Ther 2000; 80: 896-903

18. Berg K, Wood-Dauphinee S, Williams JI. The Balance Scale: reliability assessment with elderly residents and patients with an acute stroke. Scand J Rehabil Med 1995; 27: 27-36

19. Avni N, Avni I, Barenboim E, Azaria B, Zadok D, Kohen-raz R, Morad Y. Brief posturographic test as an indicator of fatigue. Psychiatry Clin Neurosci 2006; 60: 340-346

20. Kohen-Raz R. Application of tetra-ataxiametric posturography in clinical and developmental diagnosis.
Percept Mot Skill 1991; 73: 635-656

21. Yoshida S, Aoyagi K, Felson DT, Aliabadi P, Shindo H, Takemoto T. Comparison of the prevalence of radiographic osteoarthritis of the knee and hand between Japan and the United States. J Rheumatol 2002; 29: 1454-1458

22. Gardner MM, Robertson MC, Campbell AJ. Exercise in preventing falls and fall related injuries in older people: a review of randomized controlled trials. $\mathrm{Br} \mathrm{J}$ Sports Med 2000; 34: 7-17

23. Barnett A, Smith B, Lord SR, Williams M, Baumand A. Community-based group exercise improves balance and reduces falls in at-risk older people: a randomized controlled trial. Age Ageing 2003; 32: 407-414 